\title{
On fractional metric dimension of comb product graphs
}

\author{
Suhadi Wido Saputro ${ }^{1, *}$, Andrea Semaničová-Feňovč́́ková ${ }^{2}$, Martin Bača $^{2}$, Marcela Lascsáková ${ }^{2}$ \\ ${ }^{1}$ Department of Mathematics, Institut Teknologi Bandung, Indonesia \\ ${ }^{2}$ Department of Applied Mathematics and Informatics, Techical University, Košice, Slovakia
}

\begin{abstract}
A vertex $z$ in a connected graph $G$ resolves two vertices $u$ and $v$ in $G$ if $d_{G}(u, z) \neq d_{G}(v, z)$. A set of vertices $R_{G}\{u, v\}$ is a set of all resolving vertices of $u$ and $v$ in $G$. For every two distinct vertices $u$ and $v$ in $G$, a resolving function $f$ of $G$ is a real function $f: V(G) \rightarrow[0,1]$ such that $f\left(R_{G}\{u, v\}\right) \geq 1$. The minimum value of $f(V(G))$ from all resolving functions $f$ of $G$ is called the fractional metric dimension of $G$. In this paper, we consider a graph which is obtained by the comb product between two connected graphs $G$ and $H$, denoted by $G \triangleright_{o} H$. For any connected graphs $G$, we determine the fractional metric dimension of $G \triangleright_{o} H$ where $H$ is a connected graph having a stem or a major vertex.
\end{abstract}

Keywords Comb Product, Fractional Metric Dimension, Resolving Function

AMS 2010 subject classifications 05C12, 05C76, 05C78

DOI: $10.19139 /$ soic.v6i1.473

\section{Introduction}

Throughout this paper, all graphs are finite, simple, and undirected. The distance between two vertices $u, v \in V(G)$, denoted by $d_{G}(u, v)$, is the length of a shortest path from $u$ to $v$ in $G$. For $u, v \in V(G)$, we define $R_{G}\{u, v\}=\{z \in V(G) \mid d(u, z) \neq d(v, z)\}$. A vertex set $W \subseteq V(G)$ is called a resolving set of $G$ if $W \cap R_{G}\{u, v\} \neq \emptyset$ for any two distinct vertices $u, v \in V(G)$. The minimum cardinality of all resolving sets of $G$ is called the metric dimension of $G$.

The metric dimension problems were first studied by Harary and Melter [10], and independently by Slater [14]. Slater considered the minimum resolving set of a graph as the location of the placement of a minimum number of sonar/loran detecting devices in a network. So, the position of every vertex in the network can be uniquely described in terms of its distances to the devices in the set. Applications of metric dimension problem can also be found in network and verification [4], robot navigation [11], combinatorial optimization [13], pharmaceutical chemistry [5], and strategies for the mastermind game [6].

For a function $f$ defined on $V(G)$ and $W \subseteq V(G)$, let $f(W)=\sum_{w \in W} f(w)$. For every two distinct vertices $u$ and $v$ in $G$, a resolving function $f$ of $G$ is a real functions $f: V(G) \rightarrow[0,1]$ such that $f\left(R_{G}\{u, v\}\right) \geq 1$. The fractional metric dimension of $G$, denoted by $\operatorname{dim}_{f}(G), \operatorname{is} \min \{f(V(G)) \mid f$ is a resolving function of $G\}$.

Currie and Oellermann [7] defined fractional metric dimension as the optimal solution of the linear relaxation of the integer programming problem. A formulation of fractional metric dimension as a linear programming problem can be seen in [8]. The fractional metric dimension in graphs was officially initiated by Arumugam and Mathew [1] in 2012. They provided a sufficient condition for a connected graph $G$ whose fractional metric dimension is $\frac{|V(G)|}{2}$. Furthermore, Arumugam et al. [2] also characterized all graphs $G$ where $\operatorname{dim}_{f}(G)=\frac{|V(G)|}{2}$. They also

\footnotetext{
*Correspondence to: Suhadi Wido Saputro (Email: suhadi@math.itb.ac.id). Department of Mathematics, Institut Teknologi Bandung, J1. Ganesa 10 Bandung 40132 Indonesia.
}

ISSN 2310-5070 (online) ISSN 2311-004X (print)

Copyright (C) 2018 International Academic Press 
determined $\operatorname{dim}_{f}(G)$ where $G$ is Petersen graph, cycles, hypercubes, stars, wheels, friendship graph, and grids [1]. Meanwhile, the fractional metric dimension of trees and unicyclic graphs can be seen in [12].

Some authors applied this topic to some product graphs. Feng et al. [9] have determined the fractional metric dimension of Cartesian product of two graphs. Eunjeong Yi [15] investigated the fractional metric dimension of permutation graphs.

Here, we study the fractional metric dimension of comb product of connected graphs $G$ and $H$. In chemistry [3], some classes of chemical graphs can be considered as the comb product graphs. Let $G$ and $H$ be two connected graphs. Let $o$ be a vertex of $H$. The comb product between $G$ and $H$, denoted by $G \triangleright_{o} H$, is a graph obtained by taking one copy of $G$ and $|V(G)|$ copies of $H$ and identify the $i$-th copy of $H$ at the vertex $o$ with the $i$-th vertex of $G$. By the definition of comb product, we can say that $V\left(G \triangleright_{o} H\right)=\{(a, v) \mid a \in V(G), v \in V(H)\}$ and $(a, v)(b, w) \in E\left(G \triangleright_{o} H\right)$ whenever $a=b$ and $v w \in E(H)$, or $a b \in E(G)$ and $v=w=o$. We consider two vertices $a \in V(G)$ and $o \in V(H)$. We define $H(a)=\{(a, v) \mid v \in V(H)\}$ and $G(o)=\{(v, o) \mid v \in V(G)\}$.

In this paper, we study the fractional metric dimension of $G \triangleright_{o} H$ where $G$ is a connected graph and $H$ is a connected graph having a stem or a major vertex. Let $v$ be a vertex of $G$. A branch of $G$ at $v$ is defined as a maximal subgraph of $G$ which is isomorphic to a tree and containing $v$ as an end point. So, if degree of $v$ is $k$, then $v$ has at most $k$ different branches. We recall that the degree of a vertex $v$ in $G$, denoted by $\operatorname{deg}_{G}(v)$, is the number of adjacent vertices to $v$ in $G$. A branch of $v$ which is isomorphic to a path is called a path branch of $v$. If $v$ contains at least two path branches, then $v$ is called a stem of $G$. If a stem $v$ contains at least three path branches, then $v$ is called a major vertex of $G$.

\section{Preliminary results}

Let $G$ be a connected graph having a stem vertex. Let the vertex $v \in V(G)$ be a stem in $G$. Let $\mathcal{A}(v)$ be the vertex set of all vertices in path branches of a stem $v$.

\section{Lemma 1}

Let $G$ be a connected graph and $v$ is a stem in $G$. Let $f$ be a resolving function of $G$. If $v$ has $k \geq 2$ path branches, then $f(\mathcal{A}(v)) \geq \frac{k}{2}$.

Proof

Let $B_{1}(v), B_{2}(v), \ldots, B_{k}(v)$ be $k$ distinct path branches of $v$. We distinguish two cases.

1. $k=2$

Let $x \in V\left(B_{1}(v)\right)$ and $y \in V\left(B_{2}(v)\right)$ where $d_{G}(x, v)=d_{G}(y, v)$. Note that $R_{G}\{x, y\}=V\left(B_{1}(v)\right) \cup$ $V\left(B_{2}(v)\right) \subseteq \mathcal{A}(v)$. Since $f$ is a resolving function of $G$, we obtain that $f(\mathcal{A}(v)) \geq f\left(R_{G}\{x, y\}\right) \geq 1=\frac{k}{2}$.

2. $k \geq 3$

For distinct integers $i, j \in\{1,2, \ldots, k\}$, let $x_{i} \in V\left(B_{i}(v)\right)$ and $x_{j} \in V\left(B_{j}(v)\right)$ such that $d_{G}\left(x_{i}, v\right)=$ $d_{G}\left(x_{j}, v\right)$. Note that $R_{G}\left\{x_{i}, x_{j}\right\}=V\left(B_{i}(v)\right) \cup V\left(B_{j}(v)\right)$. Without loss of generality, let $f\left(V\left(B_{1}(v)\right)\right)=p$ and $f\left(V\left(B_{2}(v)\right)\right) \geq 1-p$. So, for $t \geq 3$ we have $f\left(V\left(B_{t}(v)\right)\right) \geq \max \{p, 1-p\}$. We distinguish two cases.

- $f\left(V\left(B_{t}(v)\right)\right) \geq p$

So, we have $p \geq \frac{1}{2}$. Then we obtain

$$
\begin{aligned}
f & \left(V\left(B_{1}(v)\right)\right)+f\left(V\left(B_{2}(v)\right)\right)+f\left(V\left(B_{3}(v)\right)\right)+\ldots+f\left(V\left(B_{k}(v)\right)\right) \\
& \geq p+(1-p)+p+\ldots+p \\
& \geq 1+(k-2) p \\
& \geq \frac{k}{2} \\
\text { - } f\left(V\left(B_{t}(v)\right)\right) \geq 1- & p
\end{aligned}
$$


So, we have $0 \leq p \leq \frac{1}{2}$. Then we obtain

$$
\begin{aligned}
& f\left(V\left(B_{1}(v)\right)\right)+f\left(V\left(B_{2}(v)\right)\right)+f\left(V\left(B_{3}(v)\right)\right)+\ldots+f\left(V\left(B_{k}(v)\right)\right) \\
& \quad \geq p+(1-p)+(1-p)+\ldots+(1-p) \\
& \quad \geq k-1-(k-2) p \\
& \quad \geq \frac{k}{2}
\end{aligned}
$$

From two cases above, it follows that $f(\mathcal{A}(v)) \geq f\left(V\left(B_{1}(v)\right)\right)+f\left(V\left(B_{2}(v)\right)\right)+\ldots+f\left(V\left(B_{k}(v)\right)\right) \geq \frac{k}{2}$.

By the proof of Lemma 1, if a stem $v$ in $G$ has $k \geq 2$ path branches and $f$ is a resolving function of $G$, then there exists $k-1$ path branches of a stem $v$ such that every path branch $Z$ of them satisfies $f(V(Z)) \geq \frac{1}{2}$. In lemma below, we give an existence of a resolving function $f$ of $G$ such that for every path branch $Z$ of a stem $v$ in $G$, $f(V(Z))=\frac{1}{2}$.

\section{Lemma 2}

Let $G$ be a connected graph and $v$ be a stem in $G$. Let $B_{1}(v), B_{2}(v), \ldots, B_{k}(v)$ be $k$ different path branches of $v$. Then there exists a resolving function $f$ of $G$ such that for $1 \leq i \leq k, f\left(V\left(B_{i}\right)\right)=\frac{1}{2}$.

Proof

Suppose that $g$ is a resolving function of $G$ satisfying Lemma 1 where there exists $i \in\{1,2, \ldots, k\}$ such that $g\left(V\left(B_{i}(v)\right)\right) \neq \frac{1}{2}$. Note that there exists $i \in\{1,2, \ldots, k\}$ such that $g\left(V\left(B_{i}(v)\right)\right)>\frac{1}{2}$. Now, we define a function $f: V(G) \rightarrow[0,1]$ as follow. For $w \in V(G)$,

$$
f(w)= \begin{cases}g(w), & \text { if } w \notin \mathcal{A}(v), \\ \frac{1}{2}, & \text { if the vertex } w \in \mathcal{A}(v) \text { has degree } 1, \\ 0, & \text { otherwise. }\end{cases}
$$

We will show that $f$ is a resolving function of $G$.

For $1 \leq i \leq k$, let $b_{i}$ be a leaf in $B_{i}(v)$. Let $x$ and $y$ be two distinct vertices in $G$. If $v \in R_{G}\{x, y\}$, then for $1 \leq i \leq k$, we have $b_{i} \in R_{G}\{x, y\}$, which implies $f\left(R_{G}\{x, y\}\right) \geq \frac{k}{2} \geq 1$. Otherwise, we distinguish two cases.

(i) For $1 \leq i \leq k, b_{i} \notin R_{G}\{x, y\}$

Then every vertex of $\mathcal{A}(v)$ is not an element of $R_{G}\{x, y\}$. Since $g$ is a resolving function, we obtain $f\left(R_{G}\{x, y\}\right)=g\left(R_{G}\{x, y\}\right) \geq 1$.

(ii) There exists $i \in\{1,2, \ldots, k\}$ such that $b_{i} \in R_{G}\{x, y\}$

If there exists $j \in\{1,2, \ldots, k\} \backslash\{i\}$ such that $b_{j} \in R_{G}\{x, y\}$, then we have $f\left(R_{G}\{x, y\}\right) \geq f\left(b_{i}\right)+f\left(b_{j}\right)=$ 1. Otherwise, we have $x \in V\left(B_{i}(v)\right)$ and $y \notin \mathcal{A}(v)$. We define $R_{1}^{x y}$ as a subset of $R_{G}\{x, y\}$ and $\mathcal{A}(v)$. We also define $R_{2}^{x y}=R_{G}\{x, y\} \backslash R_{1}^{x y}$. Let $P_{G}(v, y)$ be a shortest path with end points $v$ and $y$ in $G$. Then there exist $j \in\{1,2, \ldots, k\} \backslash\{i\}, z \in V\left(B_{j}(v)\right)$, and $s \in V\left(P_{G}(v, y)\right)$ such that the vertex $b_{j}$ is the only vertex among $b_{1}, b_{2}, \ldots, b_{k}$ which is the element of $R_{G}\{z, s\}$. Note that $R_{2}^{z s} \subseteq R_{2}^{x y}$. Since $g$ is a resolving function of $G$, then $g\left(R_{2}^{x y}\right) \geq g\left(R_{2}^{z s}\right) \geq \frac{1}{2}$. Thus, we obtain that $f\left(R_{G}\{x, y\}\right) \geq f\left(b_{i}\right)+f\left(R_{2}^{x y}\right)=f\left(b_{i}\right)+g\left(R_{2}^{x y}\right) \geq$ 1 .

\section{Theorem 1}

Let $G$ be a connected graph containing $m$ distinct major vertices $v_{1}, v_{2}, \ldots, v_{m}$. For $1 \leq i \leq m$, let $k_{i}$ be number of path branches of the major vertex $v_{i}$. Then

$$
\operatorname{dim}_{f}(G) \geq \sum_{i=1}^{m} \frac{k_{i}}{2}
$$

Proof

This theorem is a direct consequence of Lemma 1. 


\section{Fractional metric dimension of $G \triangleright_{o} H$ where $H$ is a path}

In this section, we consider graph $H$ as a path. Note that, a path graph of order $n \geq 3$ has $n-2$ stems. We determine the exact value of $\operatorname{dim}_{f}\left(G \triangleright_{o} H\right)$ where $G$ is any connected graph and the vertex $o \in V(H)$ is a stem.

Theorem 2

Let $G$ be a connected graph. If the vertex $o$ is a stem of a path graph $P_{m}$, then

$$
\operatorname{dim}_{f}\left(G \triangleright_{o} P_{m}\right)=|V(G)| .
$$

Proof

By the definition of a comb product, every vertex $v \in G(o)$ is a stem and $H(v) \subset \mathcal{A}(v)$. Thus, a graph $G \triangleright_{o} P_{m}$ has $|V(G)|$ stems and each stem has two path branches. According to Lemmas 1, we have $\operatorname{dim}_{f}\left(G \triangleright_{o} P_{m}\right) \geq|V(G)|$.

Now, let $V\left(P_{m}\right)=\left\{p_{i} \mid 1 \leq i \leq m\right\}$ where $E\left(P_{m}\right)=\left\{p_{i} p_{i+1} \mid 1 \leq i \leq m-1\right\}$. Let the vertex $o=p_{i}$ where $i \in\{2,3, \ldots, m-1\}$. We define a function $f: V\left(G \triangleright_{o} P_{m}\right) \rightarrow[0,1]$ as follow. For $w \in V\left(G \triangleright_{o} P_{m}\right)$,

$$
f(w)= \begin{cases}\frac{1}{2}, & \text { if } \operatorname{deg}_{G \triangleright_{o} P_{m}}(w)=1, \\ 0, & \text { otherwise. }\end{cases}
$$

We will show that $f$ is a resolving function of $G \triangleright_{o} P_{m}$. Note that $f\left(V\left(G \triangleright_{o} P_{m}\right)\right)=|V(G)|$.

Let $x$ and $y$ be two different vertices in $G \triangleright_{o} P_{m}$. If there exists a vertex $a \in V(G)$ such that $(a, o) \in R_{G \triangleright_{o} P_{m}}\{x, y\}$, then $\left(a, p_{1}\right),\left(a, p_{m}\right) \in R_{G \triangleright_{o} P_{m}}\{x, y\}$ which implies $f\left(R_{G \triangleright_{o} P_{m}}\{x, y\}\right) \geq f\left(\left(a, p_{1}\right)\right)+$ $f\left(\left(a, p_{m}\right)\right)=1$. Otherwise, there exists a vertex $a \in V(G)$ such that $x=\left(a, p_{i-j}\right)$ and $y=\left(a, p_{i+j}\right)$ where $j \in\{1,2, \ldots, \min \{i-1, m-i\}\}$. Note that $R_{G \triangleright_{o} P_{m}}\{x, y\}=\mathcal{A}((a, o))$. Then we have $f\left(R_{G \triangleright_{o} P_{m}}\{x, y\}\right)=$ $f\left(\left(a, p_{1}\right)\right)+f\left(\left(a, p_{m}\right)\right)+f\left(\mathcal{A}((a, o)) \backslash\left\{\left(a, p_{1}\right),\left(a, p_{m}\right)\right\}\right)=\frac{1}{2}+\frac{1}{2}+0=1$. Therefore, $f$ is a resolving function of $G \triangleright_{o} P_{m}$.

Let us consider $G \triangleright_{o} P_{m}$ where the vertex $o$ is not a leaf of a path graph $P_{m}$ above. Although the graph $G$ does not contain any stem vertex, but in $G \triangleright_{o} P_{m}$, all corresponding vertices from $G$ are stems. This property does not automatically occur when the vertex $o$ is a leaf of $P_{m}$.

Let $G$ be a graph of order at least three containing a vertex of degree one. Let $u, v \in V(G)$ where $\operatorname{deg}_{G}(u)=1$ and $u v \in E(G)$. Thus it is clear that the vertex $u$ is not a stem, but the vertex $v$ maybe a stem. Note that if the vertex $o$ is a leaf of $P_{m}$, then a vertex in $G \triangleright_{o} P_{m}$ which is corresponding to $u$, is also not a stem. But the corresponding vertex to $v$ in $G \triangleright_{o} P_{m}$ is a stem of $G \triangleright_{o} P_{m}$. Therefore, if the vertex $o$ is a leaf of $P_{m}$, we will only consider a connected graph $G$ where every vertex of $G$ has degree at least two, because in this case every vertex of $G$ is not a stem and its corresponding vertex in $G \triangleright_{o} P_{m}$ is also not a stem.

Now, let $G$ be a connected graph of order $n$ where every vertex of $G$ has degree at least two. Let $V(G)=$ $\left\{v_{1}, v_{2}, \ldots, v_{n}\right\}$. Let us consider the comb product graph $G \triangleright_{o} P_{m}$ where the vertex $o$ is a leaf of $P_{m}$. For two distinct vertices $v_{i}, v_{j} \in V(G)$, let $x_{i} \in P_{m}\left(v_{i}\right)$ and $x_{j} \in P_{m}\left(v_{j}\right)$. We define the following vertex sets.

- $A\left\{x_{i}, x_{j}\right\}=\left\{(z, o) \mid d_{G \triangleright_{o} P_{m}}\left(x_{i},(z, o)\right)=d_{G \triangleright_{o} P_{m}}\left(x_{j},(z, o)\right), z \in V(G) \backslash\left\{v_{i}, v_{j}\right\}\right\}$

- $\gamma\left\{x_{i}, x_{j}\right\}=\left\{z \in V(G) \mid(z, o) \in G(o) \backslash A\left\{x_{i}, x_{j}\right\}\right\}$

Note that, by the definition above, if $x_{i}=\left(v_{i}, o\right)$ and $x_{j}=\left(v_{j}, o\right)$, then $\gamma\left\{x_{i}, x_{j}\right\}=R_{G}\left\{v_{i}, v_{j}\right\}$. We also define $\gamma=\min \left\{\left|\gamma\left\{x_{i}, x_{j}\right\}\right| \mid x_{i} \in P_{m}\left(v_{i}\right), x_{j} \in P_{m}\left(v_{j}\right), v_{i}, v_{j} \in V(G)\right\}$. Thus, it is clear that $\gamma \in\{2,3, \ldots,|V(G)|\}$.

\section{Theorem 3}

Let $G$ be a connected graph where every vertex of $G$ has degree at least two. If the vertex $o$ is a leaf of a path graph $P_{m}$, then

$$
\operatorname{dim}_{f}\left(G \triangleright_{o} P_{m}\right) \leq \frac{|V(G)|}{\gamma} .
$$

Proof

Let $V\left(P_{m}\right)=\left\{p_{i} \mid 1 \leq i \leq m\right\}$ and $E\left(P_{m}\right)=\left\{p_{i} p_{i+1} \mid 1 \leq i \leq m-1\right\}$. Let the vertex $o=p_{1}$. Now, we define 
a vertex labeling $f: V(G) \rightarrow[0,1]$ such that for $(a, b) \in V\left(G \triangleright_{o} P_{m}\right)$,

$$
f((a, b))= \begin{cases}\frac{1}{\gamma}, & \text { if } b=p_{m} \\ 0, & \text { otherwise. }\end{cases}
$$

Note that $f(V(G))=\frac{|V(G)|}{\gamma}$. Now, we will show that $f$ is a resolving function of $G \triangleright_{o} P_{m}$.

Let $x$ and $y$ be two distinct vertices in $G \triangleright_{o} P_{m}$. If there exists $v \in V(G)$ such that $x, y \in P_{m}(v)$, then for every $v \in V(G),\left(v, p_{m}\right) \in R_{G \triangleright_{o} P_{m}}\{x, y\}$. Therefore, we obtain

$$
f\left(R_{G \triangleright_{o} P_{m}}\{x, y\}\right) \geq \sum_{v \in V(G)} f\left(\left(v, p_{m}\right)\right)=\frac{|V(G)|}{\gamma} \geq 1 .
$$

Now, we assume that $x \in P_{m}(v)$ and $y \in P_{m}(w)$ where $v$ and $w$ are two different vertices in $G$. Since $\left(v, p_{m}\right),\left(w, p_{m}\right) \in R_{G \triangleright_{o} P_{m}}\{x, y\}$, so we obtain

$$
f\left(R_{G \triangleright_{o} P_{m}}\{x, y\}\right)=\sum_{v \in \gamma\{x, y\}} f\left(\left(v, p_{m}\right)\right)=\frac{|\gamma\{x, y\}|}{\gamma} \geq \frac{|\gamma\{x, y\}|}{|\gamma\{x, y\}|}=1 .
$$

In Theorem 4, we give an example of a graph $G$ such that the fractional metric dimension of $G \triangleright_{o} P_{m}$ where the vertex $o$ is a leaf of $P_{m}$, is equal to the upper bound in Theorem 3 above.

Theorem 4

Let the vertex $o$ be a leaf of a path graph $P_{m}$. There exists a connected graph $G$ where every vertex of $G$ has degree at least two, such that

$$
\operatorname{dim}_{f}\left(G \triangleright_{o} P_{m}\right)=\frac{|V(G)|}{\gamma} .
$$

Proof

Let $G$ be a complete graph of order $n \geq 3$ with $V(G)=\left\{v_{1}, v_{2}, \ldots, v_{n}\right\}$. Let $V\left(P_{m}\right)=\left\{p_{1}, p_{2}, \ldots, p_{m}\right\}$ and $E\left(P_{m}\right)=\left\{p_{i} p_{i+1} \mid 1 \leq i \leq m-1\right\}$. Let the vertex $o=p_{1}$. Note that, in $G \triangleright_{o} P_{m}$, for $1 \leq i<j \leq$ $n$ and $k, l \in\{1,2, \ldots, m\}$ with $k \neq l$, we have $\left|\gamma\left\{\left(v_{i}, p_{k}\right),\left(v_{j}, p_{k}\right)\right\}\right| \leq\left|\gamma\left\{\left(v_{i}, p_{k}\right),\left(v_{j}, p_{l}\right)\right\}\right|$. Therefore, $\gamma=$ $\left|\gamma\left\{\left(v_{i}, p_{k}\right),\left(v_{j}, p_{k}\right)\right\}\right|=2$. Now, we will show that $\operatorname{dim}_{f}\left(G \triangleright_{o} P_{m}\right)=\frac{n}{2}$. According to Theorem 3, we only need to show that $\operatorname{dim}_{f}\left(G \triangleright_{o} P_{m}\right) \geq \frac{n}{2}$.

Let $f$ be a resolving function of $G \triangleright_{o} P_{m}$. For $1 \leq i<j \leq n$ and $k \in\{1,2, \ldots, m\}$, let us consider two distinct vertices $\left(v_{i}, p_{k}\right)$ and $\left(v_{j}, p_{k}\right)$. It is easy to see that $R_{G \triangleright_{o} P_{m}}\left\{\left(v_{i}, p_{k}\right),\left(v_{j}, p_{k}\right)\right\}=P_{m}\left(v_{i}\right) \cup P_{m}\left(v_{j}\right)$. Therefore, $f\left(R_{G \triangleright_{o} P_{m}}\left\{\left(v_{i}, p_{k}\right),\left(v_{j}, p_{k}\right)\right\}\right)=f\left(P_{m}\left(v_{i}\right)\right)+f\left(P_{m}\left(v_{j}\right)\right) \geq 1$. We define

$$
\alpha=\sum_{i, j \in\{1,2, \ldots, n\} \text { and } i \neq j} f\left(R_{G \triangleright{ }_{o} P_{m}}\left\{\left(v_{i}, p_{k}\right),\left(v_{j}, p_{k}\right)\right\}\right) .
$$

Since $i$ and $j$ are two different integers from $1,2, \ldots, n$, then we obtain that

$$
\alpha \geq\left(\begin{array}{l}
n \\
2
\end{array}\right)=\frac{n(n-1)}{2} .
$$

In other hand, to obtain the value of $\alpha$, every label of a vertex of $G \triangleright_{o} P_{m}$ is counted $n-1$ times. Therefore, we have a formula that

$$
\alpha=(n-1) \cdot f\left(V\left(G \triangleright_{o} P_{m}\right)\right) .
$$

Combining (1) and (2), we have $f\left(V\left(G \triangleright_{o} P_{m}\right)\right) \geq \frac{n}{2}$. 


\section{Fractional metric dimension of $G \triangleright_{o} H$ where $H$ contains a major vertex}

First, in this section we provide some properties for a resolving set between two different vertices in a comb product graph $G \triangleright_{o} H$.

Lemma 3

Let $G$ and $H$ be connected graphs. Let $o$ be a vertex of $H$. For $u, v \in V(H)$,

(i) if $o \in R_{H}\{u, v\}$, then for every two distinct vertices $a, b \in V(G)$, we have $H(b) \subset R_{G \triangleright_{o} H}\{(a, u),(a, v)\}$.

(ii) if $o \notin R_{H}\{u, v\}$, then for every vertex $b \in V(G) \backslash\{a\}$, all vertices in $H(b)$ are not elements of $R_{G \triangleright \triangleright_{o} H}\{(a, u),(a, v)\}$.

Proof

Let $o \in R_{H}\{u, v\}$. So, it is clear that $d_{H}(o, u) \neq d_{H}(o, v)$. It follows that for $a \in V(G), d_{G \triangleright_{o} H}((a, o),(a, u)) \neq$ $d_{G \triangleright_{o} H}((a, o),(a, v))$. Let $b \in V(G) \backslash\{a\}$ and $z \in H(b)$. Then

$$
\begin{aligned}
d_{G \triangleright_{o} H}(z,(a, u)) & =d_{G \triangleright_{o} H}(z,(b, o))+d_{G \triangleright_{o} H}((b, o),(a, o))+d_{G \triangleright_{o} H}((a, o),(a, u)) \\
& \neq d_{G \triangleright_{o} H}(z,(b, o))+d_{G \triangleright_{o} H}((b, o),(a, o))+d_{G \triangleright_{o} H}((a, o),(a, v)) \\
& =d_{G \triangleright_{o} H}(z,(a, v)) .
\end{aligned}
$$

Therefore, $z \in R_{G \triangleright{ }_{o} H}\{(a, u),(a, v)\}$.

Let $o \notin R_{H}\{u, v\}$. So, it is clear that $d_{H}(o, u)=d_{H}(o, v)$. It follows that for $a \in V(G), d_{G \triangleright_{o} H}((a, o),(a, u))=$ $d_{G \triangleright_{o} H}((a, o),(a, v))$. Thus, $(a, o) \notin R_{G \triangleright_{o} H}\{(a, u),(a, v)\}$. Let $b \in V(G) \backslash\{a\}$ and $z \in H(b)$. Then

$$
\begin{aligned}
d_{G \triangleright_{o} H}(z,(a, u)) & =d_{G \triangleright_{o} H}(z,(b, o))+d_{G \triangleright_{o} H}((b, o),(a, o))+d_{G \triangleright_{o} H}((a, o),(a, u)) \\
& =d_{G \triangleright_{o} H}(z,(b, o))+d_{G \triangleright_{o} H}((b, o),(a, o))+d_{G \triangleright_{o} H}((a, o),(a, v)) \\
& =d_{G \triangleright_{o} H}(z,(a, v)) .
\end{aligned}
$$

Therefore, $z \notin R_{G \triangleright_{o} H}\{(a, u),(a, v)\}$.

\section{Lemma 4}

Let $G$ and $H$ be connected graphs. Let $a$ and $b$ be two different vertices of $G$ and $o, u, v \in V(H)$. Then $(a, o) \in R_{G \triangleright_{o} H}\{(a, u),(b, v)\}$ or $(b, o) \in R_{G \triangleright_{o} H}\{(a, u),(b, v)\}$.

\section{Proof}

If $(a, o) \in R_{G \triangleright_{o} H}\{(a, u),(b, v)\}$, then the lemma is complete. Now, we assume that $(a, o) \notin$ $R_{G \triangleright_{o} H}\{(a, u),(b, v)\}$. Thus, we have $d_{G \triangleright_{o} H}((a, o),(a, u))=d_{G \triangleright_{o} H}((a, o),(b, v))=d_{G \triangleright_{o} H}((a, o),(b, o))+$ $d_{G \triangleright_{o} H}((b, o),(b, v))$. It follows that $d_{G \triangleright_{o} H}((b, o),(a, u))>d_{G \triangleright_{o} H}((b, o),(b, v))$, which implies $(b, o) \in$ $R_{G \triangleright_{o} H}\{(a, u),(b, v)\}$.

By Lemma 4, without loss of generality, let $(a, o) \in R_{G \triangleright_{o} H}\{(a, u),(b, v)\}$. If $d_{G \triangleright_{o} H}((a, o),(a, u))>$ $d_{G \triangleright_{o} H}((a, o),(b, v))$, then we also obtain $d_{G \triangleright_{o} H}((b, o),(a, u))=d_{G \triangleright_{o} H}((b, o),(a, o))+d_{G \triangleright_{o} H}((a, o),(a, u))>$ $d_{G \triangleright_{o} H}((b, o),(b, v))$, which implies $(b, o) \in R_{G \triangleright_{o} H}\{(a, u),(b, v)\}$. Otherwise, we have $d_{G \triangleright_{o} H}((a, o),(a, u))<$ $d_{G \triangleright_{o} H}((a, o),(b, v))=d_{G \triangleright_{o} H}((a, o),(b, o))+d_{G \triangleright_{o} H}((b, o),(b, v))$. From both cases above, we can say that there exists a vertex $(a, o) \in R_{G \triangleright_{o} H}\{(a, u),(b, v)\}$ such that $d_{G \triangleright_{o} H}((a, o),(a, u))<d_{G \triangleright_{o} H}((a, o),(b, v))$.

\section{Lemma 5}

Let $G$ and $H$ be connected graphs. Let $a$ and $b$ be two different vertices of $G$ and $o, u, v \in V(H)$. Let $(a, o) \in$ $R_{G \triangleright_{o} H}\{(a, u),(b, v)\}$ and $d_{G \triangleright_{o} H}((a, o),(a, u))<d_{G \triangleright_{o} H}((a, o),(b, v))$. Then $H(a) \subset R_{G \triangleright_{o} H}\{(a, u),(b, v)\}$.

\section{Proof}

Let $z \in H(a) \backslash\{(a, u)\}$. If $d_{G \triangleright_{o} H}(z,(b, v))=d_{G \triangleright_{o} H}(z,(a, u))+d_{G \triangleright_{o} H}((a, u),(b, v))$, then it is clear that $z \in R_{G \triangleright_{o} H}\{(a, u),(b, v)\}$. Now, we assume that $d_{G \triangleright_{o} H}(z,(b, v)) \neq d_{G \triangleright_{o} H}(z,(a, u))+d_{G \triangleright_{o} H}((a, u),(b, v))$. 
Suppose that $z \notin R_{G \triangleright_{o} H}((a, u),(b, v))$. Let $P$ be a shortest path in $G \triangleright_{o} H$ with end points $(a, u)$ and $(a, o)$. Let $x \in V(P) \backslash\{(a, u)\}$ such that $d_{G \triangleright_{o} H}(z,(b, v))=d_{G \triangleright_{o} H}(z, x)+d_{G \triangleright_{o} H}(x,(b, v))=d_{G \triangleright_{o} H}(z, x)+$ $d_{G \triangleright_{o} H}(x,(a, o))+d_{G \triangleright_{o} H}((a, o),(b, v))$. Note that $d_{G \triangleright_{o} H}(z,(a, u)) \leq d_{G \triangleright_{o} H}(z, x)+d_{G \triangleright_{o} H}(x,(a, u))$. So, we obtain that $d_{G \triangleright_{o} H}(x,(a, o))+d_{G \triangleright_{o} H}((a, o),(b, v)) \leq d_{G \triangleright_{o} H}(x,(a, u))<d_{G \triangleright_{o} H}((a, o),(a, u))$, a contradiction. Therefore, we have $z \in R_{G \triangleright_{o} H}((a, u),(b, v))$.

Since $(a, u) \in R_{G \triangleright_{o} H}((a, u),(b, v))$, we obtain that all vertices in $H(a)$ are resolving vertices of $(a, u)$ and $(b, v)$.

From Lemmas 4 and 5, we obtain the corollary below.

\section{Corollary 1}

Let $G$ and $H$ be connected graphs. Let $a$ and $b$ be two different vertices of $G$ and $o, u, v \in V(H)$. Then $H(a) \subset R_{G \triangleright_{o} H}\{(a, u),(b, v)\}$ or $H(b) \subset R_{G \triangleright_{o} H}\{(a, u),(b, v)\}$.

Let $v$ be a major vertex of $G$. We define $\mathcal{B}(v)$ as a vertex set of all path branches of $G$ at the major vertex $v$, including $v$.

Let $o \in V(H)$. In the case if there exists a major vertex $v$ of $H$ such that the vertex $o \in \mathcal{B}(v) \backslash\{v\}$, we define $P_{v}(o)$ as a path branch of $v$ in $H$ containing $o$. For $a \in V(G)$, we also define a vertex set $P_{v}(a, o)=\{(a, e) \mid e \in$ $\left.V\left(P_{v}(o)\right) \backslash\{v\}\right\} \subset V\left(G \triangleright_{o} H\right)$.

\section{Lemma 6}

Let $G$ and $H$ be connected graphs. Let $o \in V(H)$ such that there exists a major vertex $v$ in $H$ satisfying $o \in \mathcal{B}(v) \backslash\{v\}$. Then, there exists a resolving function $f$ of $G \triangleright_{o} H$ such that for every $a \in V(G)$ and $b \in$ $V\left(P_{v}(o)\right) \backslash\{v\}$, we have $f((a, b))=0$. Moreover, $f(H(a)) \geq \operatorname{dim}_{f}(H)-\frac{1}{2}$.

Proof

Let $f$ be a resolving function of $G \triangleright_{o} H$ such that $f((a, b))>0$. We define a vertex set $Z(a)=H(a) \backslash P_{v}(a, o)$. Note that an induced subgraph of $G \triangleright_{o} H$ by $Z(a)$, contains a stem $v$. According to Lemma 1 , we obtain $f(Z(a)) \geq 1$. Now, we define a function $g: V\left(G \triangleright_{o} H\right) \rightarrow[0,1]$ where for every $a \in V(G)$ and $w \in V(H)$,

$$
g((a, w))= \begin{cases}0, & \text { if } w \in V\left(P_{v}(o)\right) \backslash\{v\}, \\ f((a, w)), & \text { otherwise. }\end{cases}
$$

We will show that the function $g$ above is a resolving function of $G \triangleright_{0} H$.

Let $z_{1}, z_{2} \in V(H)$ and $a, b \in V(G)$. Let us consider $R_{G \triangleright_{o} H}\left\{\left(a, z_{1}\right),\left(b, z_{2}\right)\right\}$. We distinguish two cases.

1. Case $a \neq b$

By Corollary 1, all vertices of $H(a)$ or $H(b)$ are elements of $R_{G \triangleright_{o} H}\left\{\left(a, z_{1}\right),\left(b, z_{2}\right)\right\}$. Since $Z(a) \subset H(a)$ for every $a \in V(G)$, we have $g\left(R_{G \triangleright_{o} H}\left\{\left(a, z_{1}\right),\left(b, z_{2}\right)\right\}\right) \geq g(H(a)) \geq g(Z(a))=f(Z(a)) \geq 1$.

2. Case $a=b$

Let us consider Lemma 3. If $o \in R_{H}\left\{z_{1}, z_{2}\right\}$, then $H(a) \subset R_{G \triangleright_{o} H}\left\{\left(a, z_{1}\right),\left(b, z_{2}\right)\right\}$, which implies $g\left(R_{G \triangleright_{o} H}\left\{\left(a, z_{1}\right),\left(b, z_{2}\right)\right\}\right) \geq g(H(a)) \geq g(Z(a))=f(Z(a)) \geq 1$. Otherwise, all vertices of $H(c)$ with $c \in V(G) \backslash\{a\}$ are not elements of $R_{G \triangleright_{o} H}\left\{\left(a, z_{1}\right),\left(b, z_{2}\right)\right\}$. Since $o \notin R_{H}\left\{z_{1}, z_{2}\right\}$, we also have that $v \notin R_{H}\left\{z_{1}, z_{2}\right\}$. It follows that $(a, w) \notin R_{G \triangleright \triangleright_{0} H}\left\{\left(a, z_{1}\right),\left(b, z_{2}\right)\right\}$ for $w \in \mathcal{A}(v)$. Since $f$ is a resolving function of $G \triangleright_{o} H$, we obtain that $g\left(R_{G \triangleright_{o} H}\left\{\left(a, z_{1}\right),\left(b, z_{2}\right)\right\}\right)=f\left(R_{G \triangleright_{o} H}\left\{\left(a, z_{1}\right),\left(b, z_{2}\right)\right\}\right) \geq 1$.

By two cases above, we can see that $g$ is a resolving function of $G \triangleright_{o} H$.

For $a \in V(G)$, let $H^{\prime}(a)=H(a) \backslash P_{v}(a, o)$. So, $g(H(a))=g\left(H^{\prime}(a)\right)+g\left(P_{v}(a, o)\right)=g\left(H^{\prime}(a)\right)$. Since an induced subgraph of $G \triangleright_{o} H$ by $H^{\prime}(a)$ is isomorphic to a graph $H$ by deleting all vertices in a path branch $P_{v}(o)$ of $v$, except the vertex $v$, according to Lemma 1, we have that $g\left(H^{\prime}(a)\right) \geq \operatorname{dim}_{f}(H)-\frac{1}{2}$. Therefore, we obtain $g(H(a)) \geq \operatorname{dim}_{f}(H)-\frac{1}{2}$. 
Theorem 5

Let $G$ and $H$ be connected graphs. If there exists a major vertex $v$ in $H$ such that $o \in \mathcal{B}(v)$, then

$$
\operatorname{dim}_{f}\left(G \triangleright_{o} H\right)= \begin{cases}|V(G)| \cdot\left(\operatorname{dim}_{f}(H)-\frac{1}{2}\right), & \text { if the vertex } o \neq v \\ |V(G)| \cdot \operatorname{dim}_{f}(H), & \text { if the vertex } o=v .\end{cases}
$$

Proof

We distinguish two cases.

Case 1. The vertex $o \neq v$

By Lemma 6, we only need to show that $\operatorname{dim}_{f}\left(G \triangleright_{o} H\right) \leq|V(G)| \cdot\left(\operatorname{dim}_{f}(H)-\frac{1}{2}\right)$. Let $h$ be a resolving function of $H$ where $h(V(H))=\operatorname{dim}_{f}(H)$ and satisfying Lemma 2. Thus, we have $h\left(V\left(P_{v}(o)\right) \backslash\{v\}\right)=\frac{1}{2}$. We define a function $f: V\left(G \triangleright_{o} H\right) \rightarrow[0,1]$ where for every $a \in V(G)$ and $w \in V(H)$,

$$
f((a, w))= \begin{cases}0, & \text { if } w \in V\left(P_{v}(o)\right) \backslash\{v\}, \\ h(w), & \text { otherwise. }\end{cases}
$$

Note that $f\left(V\left(G \triangleright_{o} H\right)\right)=|V(G)| \cdot\left(\operatorname{dim}_{f}(H)-\frac{1}{2}\right)$. By using the similar argument as in the proof of Lemma 6, we obtain that $f$ is a resolving function of $G \triangleright_{o} H$.

Case 2. The vertex $o=v$

Let $h$ be a resolving function of $H$ where $h(V(H))=\operatorname{dim}_{f}(H)$. We define a function $f: V\left(G \triangleright_{o} H\right) \rightarrow[0,1]$ where for every $a \in V(G)$ and $w \in V(H), f((a, w))=h(w)$. Note that $f\left(G \triangleright_{o} H\right)=|V(G)| \cdot \operatorname{dim}_{f}(H)$. We will show that $f$ is a resolving function of $G \triangleright_{0} H$.

Let $z_{1}, z_{2} \in V(H)$ and $a, b \in V(G)$. Let us consider $R_{G \triangleright_{o} H}\left\{\left(a, z_{1}\right),\left(b, z_{2}\right)\right\}$. We distinguish two cases.

1. Case $a \neq b$

By Corollary 1, all vertices of $H(a)$ or $H(b)$ are elements of $R_{G \triangleright_{o} H}\left\{\left(a, z_{1}\right),\left(b, z_{2}\right)\right\}$. Thus, $f\left(R_{G \triangleright_{o} H}\left\{\left(a, z_{1}\right),\left(b, z_{2}\right)\right\}\right) \geq f(H(a))=\operatorname{dim}_{f}(H) \geq 1$.

2. Case $a=b$

Let us consider Lemma 3. If $o \in R_{H}\left\{z_{1}, z_{2}\right\}$, then $H(a) \subset R_{G \triangleright_{o} H}\left\{\left(a, z_{1}\right),\left(b, z_{2}\right)\right\}$, which implies $f\left(R_{G \triangleright_{o} H}\left\{\left(a, z_{1}\right),\left(b, z_{2}\right)\right\}\right) \geq f(H(a))=\operatorname{dim}_{f}(H) \geq 1$. Otherwise, all vertices of $H(c)$ with $c \in V(G) \backslash\{a\}$ are not elements of $R_{G \triangleright_{o} H}\left\{\left(a, z_{1}\right),\left(b, z_{2}\right)\right\}$. Since $o \notin R_{H}\left\{z_{1}, z_{2}\right\}$, we have that $R_{G \triangleright_{o} H}\left\{\left(a, z_{1}\right),\left(b, z_{2}\right)\right\} \subseteq H(a)$. Since $h$ is a resolving function of $H$, we obtain that $f\left(R_{G \triangleright_{o} H}\left\{\left(a, z_{1}\right),\left(b, z_{2}\right)\right\}\right)=h\left(R_{H}\left\{z_{1}, z_{2}\right\}\right) \geq 1$.

By two cases above, we obtain that $f$ is a resolving function of $G \triangleright_{o} H$.

Now, we will show that $\operatorname{dim}_{f}\left(G \triangleright_{o} H\right) \geq|V(G)| \cdot \operatorname{dim}_{f}(H)$. Suppose that $\operatorname{dim}_{f}\left(G \triangleright_{o} H\right)<|V(G)|$. $\operatorname{dim}_{f}(H)$. Let $g$ be a resolving function of $G \triangleright_{o} H$ such that $g\left(V\left(G \triangleright_{o} H\right)\right)=\operatorname{dim}_{f}\left(G \triangleright_{o} H\right)$. Then there exists a vertex $a \in V(G)$ such that $g(H(a))<\operatorname{dim}_{f}(H)$. By considering Lemmas 1 and 2, there exist two distinct vertices $z_{1}$ and $z_{2}$ in $H$ with $v \notin R_{H}\left\{z_{1}, z_{2}\right\}$ such that $g\left(R_{G \triangleright_{o} H}\left\{\left(a, z_{1}\right),\left(a, z_{2}\right)\right\}\right)<1$.

\section{Acknowledgement}

This work was partially supported by Riset Program Penelitian, Pengabdian Masyarakat, dan Inovasi (P3MI) 1016/I1.C01/PL/2017, by APVV-15-0116, and by VEGA 1/0385/17.

\section{REFERENCES}

1. S. Arumugam, and V. Mathew, The fractional metric dimension of graphs, Discrete Math., vol. 32, pp. 1584-1590, 2012.

2. S. Arumugam, V. Mathew, and J. Shen, On fractional metric dimension of graphs, Discrete Math. Algorithms Appl., vol. 5 no. 4 , $1350037,2013$.

3. M. Azari, and A. Iranmanesh, Chemical graphs constructed from rooted product and their Zagreb indices, MATCH Commun. Math. Comput. Chem., vol. 70, pp. 901-919, 2013. 
4. Z. Beerliova, F. Eberhard, T. Erlebach, and L.S. Ram, Network discovery and verification, IEEE J. Sel. Areas Commun., vol. 24, pp. 2168-2181, 2006.

5. G. Chartrand, L. Eroh, M.A. Johnson, and O.R. Oellermann, Resolvability in graphs and the metric dimension of a graph, Discrete Appl. Math., vol. 105, pp. 99-113, 2000.

6. V. Chvátal, Mastermind, Combinatorica, vol. 3, pp 325-329, 1983.

7. J. Currie, and O.R. Oellermann, The metric dimension and metric independence of a graph, J. Combin. Math. Combin. Comput., vol. 39, pp. 157-167, 2001.

8. M. Fehr, S. Gosselin, and O.R. Oellermann, The metric dimension of Cayley graphs, Discrete Math., vol. 306, pp. 31-41, 2006.

9. M. Feng, B. Lv, and K. Wang, On the fractional metric dimension of graphs, Discrete Appl. Math., vol. 170, pp. 55-63, 2014.

10. F. Harary, and R.A. Melter, On the metric dimension of a graph, Ars Combin., vol. 2, pp. 191-195, 1976.

11. S. Khuller, B. Raghavachari, and A. Rosenfeld, Landmarks in graphs, Discrete Appl. Math., vol.70, pp. 217-229, 1996.

12. D.A. Krismanto, and S.W. Saputro, Fractional metric dimension of tree and unicyclic graph, Procedia Comput. Sci., vol. 74, pp. 47-52, 2015.

13. A. Sebő, and E. Tannier, On metric generators of graphs, Math. Oper. Res., vol. 29 no.2, pp. 383-393, 2004.

14. P.J. Slater, Leaves of trees, Congr. Numer., vol. 14, pp. 549-559, 1975.

15. E. Yi, The fractional metric dimension of permutation graphs, Acta Math. Sinica, vol. 31 no. 3, pp. 367-382, 2015. 